\title{
How synonymous mutations alter enzyme structure and function over long time scales
}

Yang Jiang ${ }^{1}$, Syam Sundar Neti ${ }^{1}$, Priya Pradhan ${ }^{1}$, Squire J. Booker ${ }^{1,2,3}$ and Edward P. O’Brien ${ }^{1}$ 4,5, *

1 Department of Chemistry, Penn State University, University Park, Pennsylvania, United States

2 Department of Biochemistry and Molecular Biology, Pennsylvania State University, University Park, Pennsylvania, United States

${ }^{3}$ Howard Hughes Medical Institute, Pennsylvania State University, University Park, Pennsylvania, United States

${ }^{4}$ Bioinformatics and Genomics Graduate Program, The Huck Institutes of the Life Sciences, Penn State University, University Park, Pennsylvania, United States

5 Institute for Computational and Data Sciences, Penn State University, University Park, Pennsylvania, United States

* To whom correspondence should be addressed: epo2@psu.edu 


\begin{abstract}
The specific activity of enzymes can be altered over long time scales in cells by synonymous mutations, which change an mRNA molecule's sequence but not the encoded protein's primary structure. How this happens at the molecular level is unknown. Here, we resolve this issue by applying multiscale modeling to three $E$. coli enzymes - type III chloramphenicol acetyltransferase, $\mathrm{D}$-alanine-D-alanine ligase $\mathrm{B}$, and dihydrofolate reductase. This modeling involves coarsegrained simulations of protein synthesis and post-translational behavior, all-atom simulations as a test of robustness, and QM/MM calculations to characterize function. We first demonstrate that our model accurately predicts experimentally measured changes in specific activity due to synonymous mutations. Then, we show that changes in codon translation rates induced by synonymous mutations cause shifts in co-translational and post-translational folding pathways that kinetically partition molecules into subpopulations that very slowly interconvert to the native, functional state. These long-lived states exhibit reduced catalytic activity, as demonstrated by their increased activation energies for the reactions they carry out. Structurally, these states resemble the native state, with localized misfolding near the active sites of the enzymes. The localized misfolding involves noncovalent lasso entanglements - a topology in which the protein backbone forms a loop closed by noncovalent native contacts which is then threaded by another portion of the protein. Such entanglements are often kinetic traps, as they can require a large proportion of the protein to unfold, which is energetically unfavorable, before they disentangle and attain the native state. The near-native-like structures of these misfolded states allow them to bypass the proteostasis machinery and remain soluble, as they exhibit similar hydrophobic surface areas as the native state. These entangled structures persist in all-atom simulations as well, indicating that these conclusions are independent of model resolution. Thus, synonymous mutations cause shifts in the co- and post-translational structural ensemble of proteins, whose altered subpopulations lead to long-term changes in the specific activities of some enzymes. The formation of entangled subpopulations is therefore a mechanism through which changes in translation elongation rate alter ensemble-averaged specific activities, which can ultimately affect the efficiency of biochemical pathways and phenotypic traits.
\end{abstract}




\section{Introduction}

In both in vitro and in vivo experiments, the specific activity of protein enzymes - that is, the catalytic turnover per unit time per unit mass of soluble protein - can change depending on the codons used to encode the protein ${ }^{1,2,3,4,5,6}$. The specific activity of the $E$. coli enzyme type III chloramphenicol acetyltransferase (CAT-III), for example, decreases by approximately $20 \%$ when fast-translating synonymous mutations are introduced into its transcript ${ }^{1,}{ }^{6}$. Synonymous mutations change the sequence of nucleotides composing an mRNA molecule, which in turn changes the speed at which translation elongation occurs ${ }^{7}$ but not the protein primary structure. Specific activity measurements control for differences in protein expression and the formation of insoluble aggregates through centrifugation or gel separation ${ }^{1,2,3,8}$. For enzymes that do not require post-translational modifications, these observed changes in specific activity indicate that inside cells, newly expressed proteins can populate long-lived conformational states that are not native, have reduced functionality, somehow bypass the chaperone and degradation machinery, and do not aggregate. Furthermore, these observations indicate that the distribution of these kinetically trapped states is sensitive to changes in translation elongation speed.

This fourth state of soluble proteins is distinct from the three typical states ${ }^{9}$ of a protein being either folded and functional, misfolded and aggregated, or degraded. The structural, kinetic, and energetic properties of this fourth state of proteins is a fundamental, unanswered question in biochemistry and molecular biology. Soluble, nonfunctional states do not just occur for enzymes, other protein functions can also be altered. The hormone-transporting protein transthyretin, for example, can have $20 \%$ of its soluble fraction in a nonfunctional state ${ }^{10}$. There are hints in the literature of some of the structural properties of this fourth state. NMR-derived structures of $\beta$ gamma-crystallin produced from two synonymous mRNA variants found that it populated two conformations differing in only the formation of a disulfide bond ${ }^{11}$. Disulfide bonds represent an energetically strong constraint on folding topologies and are uncommon, only $5 \%$ and $20 \%$, respectively, of $E$. coli and human cytoplasmic proteins contain disulfide bonds ${ }^{12}$. Furthermore, many enzymes that exhibit specific activity changes due to synonymous mutations do not contain disulfide bonds $1,2,3,5$. Thus, the nature of these structurally altered subpopulations has not yet been resolved, although experimental data rule out misfolding involving quaternary structures. For example, gel separation and chromatography experiments rule out the formation of offpathway dimers and higher-order oligomers for many enzymes that normally function in a monomeric state st, 2, 4, $^{\text {. }}$.

Here, we use a novel multiscale approach across the dimensions of time, space, and energy to simulate the synthesis, post-translational behavior, and function of enzymes under different translation rate schedules that arise from synonymous codons. We first establish that our modeling approach can correctly predict the experimentally measured changes in specific activity for CAT-III and D-alanine-D-alanine ligase B (DDLB). We then apply the model to enzymes whose activity is sensitive to synonymous mutations and enzymes whose activity is not. Dissecting the structures and kinetics of co- and post-translational folding that occur in our simulations, we show that synonymous mutations shift the folding pathways and populations of near-native non-covalent lasso entanglements, each of which has its own intrinsic activity $\left(k_{\text {cat }}\right)$ and leads to long-term changes in the enzymatic specific activity.

\section{Methods}

Coarse-grained (CG) simulation model. To model transitions between the native state and the unfolded state, we utilized a Gō-based CG model ${ }^{13,14,15,16,17}$ for all the proteins studied. Briefly, this CG model represents each residue as a single interaction site centered on the Ca position 
and uses a structure-based potential energy function (see Supplementary equation (1)). The force field parameters were optimized by training the parameters to reproduce the folding stability free energies of 18 small single-domain proteins ${ }^{18}$, followed by assignment of the minimum values that can reproduce the structural stability for a given protein. To map the simulation timescale to an experimental timescale, we use the scaling factor $\alpha$, which is the ratio of bulk experimental folding times to simulated folding times (see Supplementary Table 4). The high-resolution crystal structure PDB $4 \mathrm{v} 9 \mathrm{~d}^{19}$ was used to coarse-grain the $E$. coli ribosome, with the A-and P-site tRNA molecules modeled based on the PDB structure 5 jte. ${ }^{20}$ The entire $50 \mathrm{~S}$ subunit, including the Aand P-site tRNAs, was coarse-grained using the three/four-point model of ribosomal RNA ${ }^{13}$ and the Ca model for ribosomal protein ${ }^{13}$ and then truncated to include only those CG interaction sites within $30 \AA$ of the centerline of the exit tunnel, within $20 \AA$ of the peptidyl transferase center (PTC, identified as A2602 in the 23S rRNA of the E. coli ribosome), and the interaction sites at the ribosome surface near the exit tunnel opening. This resulted in 4,577 interaction sites for the cropped CG E. coli ribosome.

Protein synthesis on the ribosome was modeled using a continuous synthesis protocol that describes A-site tRNA binding, peptidyl transfer, tRNA translocation, and ribosome trafficking ${ }^{21}$ at each nascent chain length using codon translation rates obtained from the model of Fluitt \& Viljoen $^{22}$ (see Supplementary equations (11) and (13)). Post-translational dynamics were modeled by simulating the nascent protein in the absence of the ribosome. Simulations for both co- and post-translational folding were performed via Langevin dynamics with a collision frequency of $0.05 \mathrm{ps}^{-1}$ and a time step of $15 \mathrm{fs}$ using OpenMM ${ }^{23}$. Details of the model setup can be found in Supplementary Methods Sections 1 to 8.

Virtual screening for enzymes that exhibit kinetic traps. To identify candidate enzymes that may have kinetic traps, we created a dataset of well-characterized monomeric $E$. coli enzymes by searching the relevant databases $\mathrm{EzCatDB}^{24,}{ }^{25}$, UniProt ${ }^{26}$ and RCSB PDB ${ }^{27}$. The wild-type enzymes were then parameterized, and their synthesis and post-translational dynamics were simulated with a 14-day wall time. The candidates were selected by using the scoring function

$$
\text { Score }=\frac{1}{2} \times\left[\left(1-e^{-\left\langle\tau_{F}^{\text {post }}\right)}\right)+\Theta_{\text {binding }}\right]
$$

where $\left\langle\tau_{\mathrm{F}}^{\text {post }}\right\rangle$ is the mean post-translational folding time calculated by using the double-pathway kinetics scheme of Supplementary equation (6) with no delay time (i.e., $t_{1}=t_{2}=0$ ) and $\Theta_{\text {binding }}$ is an indicator of whether (equals 1 ) or not (equals 0 ) misfolding occurs at or near the substrate binding site. A higher score indicates a higher possibility for the enzyme to have long-lived kinetic traps that have misfolding in the substrate binding pocket during its post-translational folding dynamics. Further details of the screening can be found in Supplementary Methods Section 9.

Characterizing post-translational misfolded structures. The misfolded structures of the posttranslational folding process were characterized by using the metrics $G$ and $Q_{\text {act. }} G$ is an order parameter that measures the extent to which there is a change of entanglement in a given structure compared to the native structure and is calculated as

$$
G=\frac{1}{N} \sum_{(i, j)} \Theta\left((i, j) \in n c \cap g(i, j) \neq g^{\text {native }}(i, j)\right),
$$

where $(i, j)$ is one of the native contacts in the native crystal structure; $n c$ is the set of native contacts formed in the current structure; $g(i, j)$ and $g^{\text {native }}(i, j)$ are, respectively, the total linking number of the native contact $(i, j)$ in the current and native structures estimated using 
Supplementary equation (16) (see Supplementary Methods Section 11 for details); $N$ is the total number of native contacts within the native structure; and the selection function $\Theta$ equals 1 when the condition is true and 0 when it is false. The larger $G$ is the larger the number of residues that have changed their entanglement status relative to the native state. That is $G$ reports on the presence of non-native entanglements in structures.

$Q_{\text {act }}$ is the fraction of native contacts that have formed in the enzyme substrate binding pocket. Residues composing the substrate binding pocket were identified as those residues within $8 \AA$ of any atoms of the relevant ligands present in the crystal structure. The $Q_{\text {act }}$ values were calculated for all the native contacts between one atom within the substrate binding pocket and any other atom, as shown below:

$$
Q_{\mathrm{act}}=\frac{\sum_{i \in I} \sum_{j \in J} \Theta(i, j \mid \text { Current })}{\sum_{i \in I} \sum_{j \in J} \Theta(i, j \mid \text { Native })},
$$

where $i$ and $j$ are the residue indices and satisfy $j>i+3 ; l$ is the intersection set of residues within secondary structure elements ( $\alpha$-helical or $\beta$-strands) and the substrate binding pocket; $J$ is the set of residues within secondary structure elements; $\Theta(i, j \mid$ Current $)$ and $\Theta(i, j \mid$ Native $)$ are step functions that equal 1 when residue $i$ and $j$ have native contact and 0 when $i$ and $j$ do not have native contact in the current structure and native structure, respectively. Native contacts are considered formed when the distance between the $\mathrm{C} \alpha$ atoms of residues $i$ and $j$ does not exceed 1.2 times their native distance and the native distance does not exceed $8 \AA$. In the case of CATIII, residue set $I$ also includes native contacts in the trimer interface region (residues 25 to 33 and 150 to 157) to monitor the folding of the trimer interface as well. Note that the native contacts used in estimating $Q_{\text {act }}$ are restricted to those within secondary structure elements, while the entire set of native contacts is used to estimate $G$. Details of the assessment procedure can be found in Supplementary Methods Section 12.

Specific activity estimation. For each metastable state $i$, identified using the Markov state modeling procedure reported in Supplementary Methods Section 12, we randomly select five conformations from all the microstates based on the probability distribution of the microstates within the metastable state and back-map them to all-atom structures (see Supplementary Methods Section 13). We then used QM/MM simulations (see Supplementary Methods Section 14) to calculate the transition state barrier for each of the five conformations, and from these, we determined the median activation barrier height $\Delta G_{i}^{\ddagger}$. Only the metastable states that form a nearnative active site $\left(Q_{\text {act }} \geq 0.6\right)$, as well as the native state, were taken to estimate $\Delta G_{i}^{\ddagger}$, while the others were considered having an infinite barrier height (zero reaction rate). Assuming the rate constants of each metastable state have similar pre-exponential factors that can be treated as a constant, the specific activity for a protein can be estimated by using the probability distribution of metastable states (state probability $p_{i}$ ) and the activation free energy barrier height $\left(\Delta G_{i}^{\ddagger}\right)$ of each state as

$$
\text { Specific activity }=\frac{A}{m} \sum_{i=1}^{N} p_{i} \cdot e^{-\frac{1}{R T} \Delta G_{i}^{\ddagger}},
$$

where $m$ is the molecular weight of the enzyme; $A$ is the pre-exponential factor allowing us to convert $\Delta G_{i}^{\ddagger}$ to the reaction rate constant; $N$ is the total number of metastable states; $R$ is the gas constant; and $T$ is the temperature. In many codon usage studies ${ }^{1,3,8}$, the relative specific activity is often used to compare the enzymatic activities of a protein produced by synonymous mRNA variants. In this study, the maximum specific activity among the fast and slow synonymous mRNA variants of an enzyme was used for normalization: 


$$
\left\{\begin{array}{l}
S A_{\text {fast }}^{*}=\frac{S A_{\text {fast }}}{\max \left\{S A_{\text {fast }}, S A_{\text {slow }}\right\}} \\
S A_{\text {slow }}^{*}=\frac{S A_{\text {slow }}}{\max \left\{S A_{\text {fast }}, S A_{\text {slow }}\right\}}
\end{array}\right.
$$

where $\mathrm{SA}^{*}$ is the relative specific activity. Note that the pre-exponential factor $A$ and the molecular weight $m$ cancel out when specific activity is normalized; therefore, they do not need to be estimated. The details of estimating the state probability $p_{i}$ (accounting for the soluble fraction only) and the activation free energy barrier height $\left(\Delta G_{i}^{\ddagger}\right)$ are presented in Supplementary Methods Section 14 . The materials and experimental methods for measuring the specific activity of DDLB variants are presented in Supplementary Methods Section 18.

\section{Results}

Recapitulating experimental trends of CAT-III. We first applied our modeling protocol to the $E$. coli enzyme CAT-III to test if the method accurately predicts the influence of synonymous mutations on specific activity. CAT-III has been studied experimentally and shown to have a reduced specific activity when faster-translating codons are introduced through synonymous mutations $^{1}$. We created both fast- and slow-translating synonymous mRNA sequences (denoted, respectively, CAT-III fast and CAT-IIIslow) by replacing each wild-type codon with its fastest or slowest synonymous variant (see Fig. $1 \mathrm{~b}$ and Supplementary Methods Section 19). The resulting slow-mRNA variant takes two times longer to translate than the fast variant (see Fig. 1f). We simulated the synthesis and post-translational behavior of CAT-III resulting from the fast- and slow-mRNA variants and calculated their respective specific activities (equation (4)) at the end of the post-translational simulations. In our model, CAT-III fast exhibited $81.4 \%(95 \%$ confidence interval $(\mathrm{Cl})$ : $[70.0 \%, 94.8 \%]$ from bootstrapping, $p$-value $=0.0035$ from random permutation test, $10^{6}$ permutations) of the specific activity of CAT-III slow (Fig. 1g). This comparison, known as the 'relative specific activity' (equation (5)), is common in biochemical studies ${ }^{1,3}$. A $20 \%$ decrease in activity has been observed experimentally ${ }^{1}$. Thus, our modeling approach qualitatively recapitulates experimentally observed changes in enzyme activity due to synonymous mutations. 
a

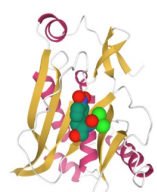

CAT-III

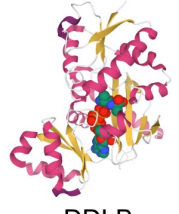

DDLB

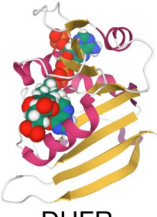

DHFR

b

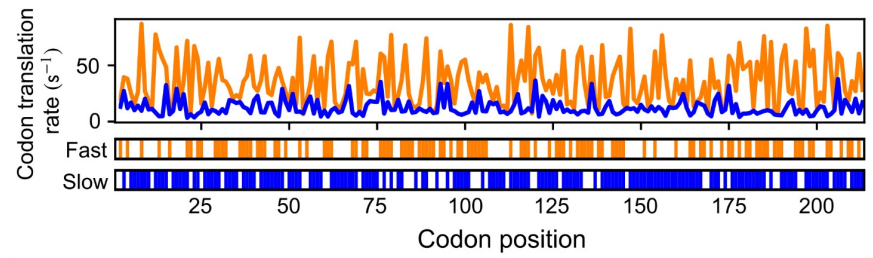

C

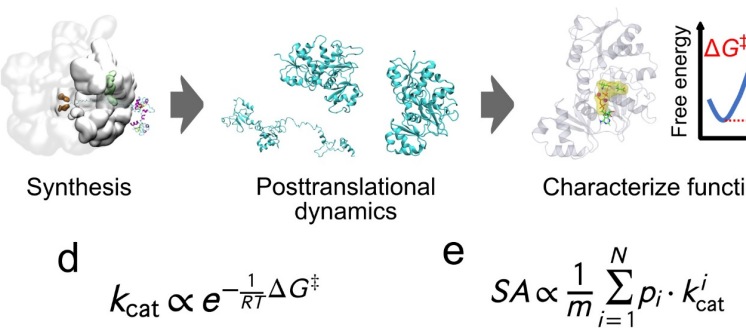

f
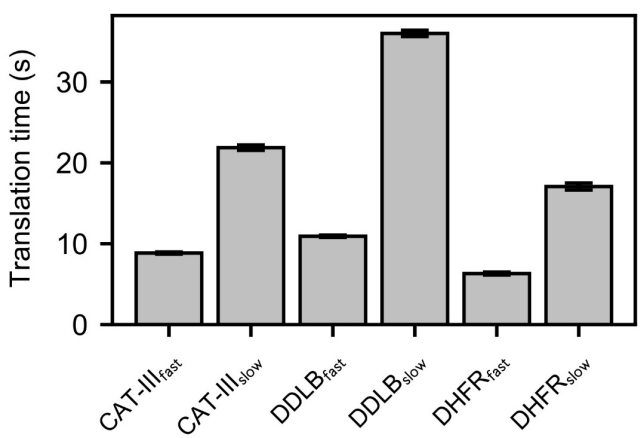

9

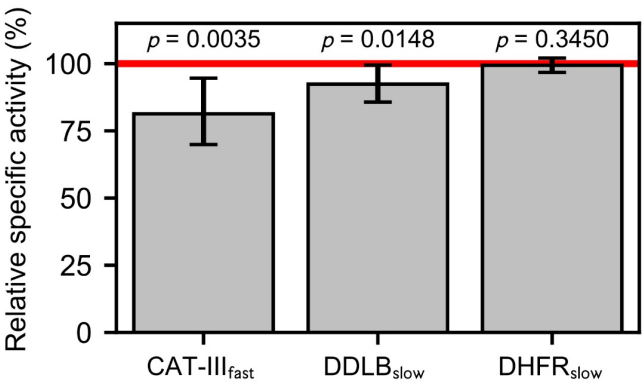

Figure 1. A multiscale approach for understanding the influence of synonymous codons on the structure and function of enzymes. a, Crystal structures of the three enzymes investigated in this study, with secondary structure elements highlighted and substrates presented; $b$, codon translation rate of CATIII fast and slow synonymous mRNA variants, with the mutation sites presented on the bottom bars; c scheme diagram of the multiscale approach, including the CG protein synthesis and post-translational dynamics, and the all-atom characterization of enzymatic functionality; d, enzymatic reaction rate $k_{\text {cat }}$ estimated from the activation free energy $\Delta G^{\ddagger}$; e, enzyme specific activity (SA) estimated as the ensemble average of reaction rates $k_{\text {cat }}$ (see equation (4)); f, comparison of the translation time for the fast and slow synonymous mRNA variants; $g$, relative SAs for CAT-III, DDLB and DHFR. The SA values are normalized to the higher SA value found in fast- and slow-mRNA variants. Error bars in panels $\mathrm{f}$ and $\mathrm{g}$ are $95 \% \mathrm{Cls}$ about the mean, which were estimated using bootstrapping. p-values characterize the difference in SAs between the proteins produced from the fast and slow variants. $p$-values were calculated using the permutation test. The SAs of CAT-III and DDLB are sensitive to translation speed changes, and that of DHFR is not.

Other enzymes that are sensitive to changes in translation speed. We next applied our model to identify other $E$. coli enzymes whose specific activities are sensitive to translation speed changes. To do this, we developed a virtual screening strategy that identifies misfolding-prone proteins exhibiting long-lived, post-translational kinetic traps (see Methods), as we hypothesized that the activity of these enzymes is more likely to be sensitive to translation speed changes. Due to the large number of $E$. coli enzymes that have an unknown catalytic mechanism or uncharacterized protein-substrate complex structures, we focused on only 14 well-characterized monomeric enzymes (Table 1). We synthesized each protein on the ribosome using the translation rate arising from their wild-type mRNA sequences, followed by release from the ribosome and a post-translational simulation phase. To identify good candidates, we scored (equation (1)) each of the 14 enzymes based on whether they misfolded near the substrate binding site and whether the misfolding events were long-lived. Porphobilinogen deaminase never exhibited any folding events during the simulations and therefore was excluded from further study. The protein with the highest score, D-alanine-D-alanine ligase B (DDLB), was selected as a candidate whose enzymatic activity we hypothesized is likely to be sensitive to translation speed 
changes, whereas the protein with the lowest score, dihydrofolate reductase (DHFR), was identified as an enzyme whose activity is likely to be insensitive to synonymous mutations.

Table 1. The 14 E. coli enzymes studied using our virtual screening strategy. $\left\langle\tau_{\mathrm{F}}^{\text {post }}\right\rangle$, calculated posttranslational mean folding time. $\Theta_{\text {binding }}$ indicates whether misfolding is (' 1 ') or is not (' 0 ') located at or near the substrate binding site. 'Score' is the scoring function calculated from equation (1).

\begin{tabular}{|c|c|c|c|c|c|}
\hline Protein Name & Length (residues) & PDB ID & $\left\langle\tau_{\mathbf{F}}^{\text {post }}\right\rangle(\mathbf{s})$ & $\boldsymbol{\Theta}_{\text {binding }}$ & Score \\
\hline D-alanine-D-alanine ligase $B(D D L B)$ & 306 & $4 \mathrm{C} 5 \mathrm{C}$ & $9.8 \times 10^{9}$ & 1 & 1.00 \\
\hline Quinone oxidoreductase 2 & 303 & $2 \mathrm{ZCV}$ & 3.9 & 1 & 0.99 \\
\hline Peptide deformylase & 169 & $3 \mathrm{~K} 6 \mathrm{~L}$ & 3.4 & 1 & 0.98 \\
\hline Phosphoenolpyruvate carboxykinase & 540 & 1AQ2 & $5.2 \times 10^{7}$ & 0 & 0.50 \\
\hline Adenylate kinase & 214 & 1AKE & 9.5 & 0 & 0.50 \\
\hline Dephospho-CoA kinase & 206 & $1 \mathrm{VHL}$ & 4.8 & 0 & 0.50 \\
\hline Protein-L-isoaspartate O-methyltransferase & 208 & 3LBF & 3.4 & 0 & 0.48 \\
\hline Methionyl-tRNA formyltransferase & 315 & 2FMT & 1.0 & 0 & 0.32 \\
\hline Phosphoribosylglycinamide formyltransferase & 212 & $1 \mathrm{C} 2 \mathrm{~T}$ & 0.65 & 0 & 0.24 \\
\hline Flavodoxin/ferredoxin--NADP reductase & 248 & 1FDR & 0.09 & 0 & 0.05 \\
\hline DNA-3-methyladenine glycosylase 2 & 282 & $3 \mathrm{CW} 7$ & 0.07 & 0 & 0.03 \\
\hline 2-Dehydropantoate 2-reductase & 303 & 2OFP & 0.05 & 0 & 0.02 \\
\hline Dihydrofolate reductase (DHFR) & 159 & $4 \mathrm{KJK}$ & 0.03 & 0 & 0.01 \\
\hline Porphobilinogen deaminase & 313 & 1PDA & - & - & - \\
\hline
\end{tabular}

Next, we simulated the synthesis and post-translational dynamics of DDLB and DHFR from their fast- and slow-translating mRNA variants (sequences are presented in Supplementary Methods Section 19) using the same simulation protocol as applied to CAT-III. The slow variants of DDLB and DHFR translate, respectively, three and two times slower than their fast variants (Fig. 1f). We found that the specific activity of DDLB slow was $92.4 \%(95 \% \mathrm{Cl}$ : $[85.7 \%, 99.3 \%])$ that of DDLB fast $_{\text {t }}$ $(p$-value $=0.0148) 60 \mathrm{~s}$ after synthesis was completed. DHFR slow exhibited a specific activity $99.4 \%$ $(95 \% \mathrm{Cl}:[96.7 \%, 102.1 \%])$ that of $\mathrm{DHFR}_{\text {fast }}$, which were not significantly different ( $p$-value = $0.3450)$. Therefore, our model describes enzymes whose specific activity is either sensitive (DDLB) or insensitive (DHFR) to changes in translation speed over long-time scales.

Accurate prediction of changes in specific activity. To experimentally test if DDLB $B_{\text {slow }}$ has a reduced enzymatic activity compared to $D D L B_{\text {fast }}$ we recombinantly expressed the fast and slow DDLB variants in $E$. coli using the same mRNA sequences as in the simulations, purified the enzyme, and then assayed the reaction kinetics (see Supplementary Methods Section 18). We observe that the fast variant had a higher level of protein expression 5 hours after induction than the slow variant, consistent with the fast mRNA variant translating faster (Supplementary Fig. 12). The reaction rate constant $k_{\mathrm{cat}}$ was measured across five biological replicates of the fast and slow variants (see Supplementary table 11). We find the specific activity of DDLB slow is, on average, $88 \%\left(\left\langle k_{\text {cat }}^{\text {slow }} / k_{\text {cat }}^{\text {fast }}\right\rangle, 95 \% \mathrm{Cl}\right.$ : [81.3\%, 94.8\%]) that of $\operatorname{DDLB}_{\text {fast }}(p$-value $=0.0186$, one-tailed t-test for $\left.\left\langle k_{\text {cat }}^{\text {slow }} / k_{\text {cat }}^{\text {fast }}\right\rangle<1\right)$. Thus, our modeling approach successfully predicts changes in activity for DDLB and CAT-III, indicating it is accurate. 
Near-native, lasso-like entangled structures are populated by the three enzymes. Next, in our simulations we identified the structures, catalytic properties, and folding pathways that cause these activity changes. We first examined the structural distributions of CAT-III, DDLB and DHFR in the post-translational simulations by using a clustering algorithm that uses both structural information and temporal interconversion rates between metastable states. Specifically, after numerous tests of different metrics, we structurally clustered on the basis of the fraction of native contacts formed in substrate binding regions (denoted as Qact, equation (3)) and the fraction of native contacts that exhibit non-native topological entanglements (denoted as $G$, equation (2), see Methods for description). Microstates were identified by clustering the post-translational trajectories of both synonymous variants together for each protein using the $k$-means algorithm ${ }^{28}$, and then the microstates were coarse-grained to a smaller number of metastable states using the PCCA+ algorithm ${ }^{29}$ (see Fig. 2a and b for CAT-III, Fig. 3a and b for DDLB and Fig. $4 a$ and $b$ for DHFR).

Across the metastable states, we observed diverse misfolded structures for CAT-III (see Supplementary Fig. 5) and DDLB (see Supplementary Fig. 6). Most of the misfolded structures exhibited topological entanglements, and many of these entangled structures were near native ( $Q_{\text {act }} \geq 0.6$ and $G \geq 0.02$; interactive visualization of some entangled structures is provided on the supplementary website https://yuj179.github.io/topo entanglements/). Misfolded, metastable states P9, P10, P11, P12 and P13 of CAT-III exhibited $Q_{\text {act }}$ and $G$ values of $(0.63,0.21),(0.64$, $0.05),(0.79,0.02),(0.87,0.13)$, and $(0.85,0.09)$, respectively, while the native state $(P 14)$ had values of $(0.87,0.00)$ (Fig. $2 \mathrm{~h}$ and Supplementary Fig. 5$)$. The DDLB misfolded states P4, P5, P6, P8, P9, P10 and P11 exhibited values of $(0.62,0.13),(0.66,0.06),(0.62,0.09),(0.85,0.20),(0.88$, $0.08),(0.85,0.04)$ and $(0.96,0.14)$, respectively, while the native state $(P 12)$ had values of $(0.93$, 0.01) (Fig. 3h and Supplementary Fig. 6). In contrast, DHFR exhibited fewer misfolded states and only one entangled, misfolded state, with $Q_{\text {act }}$ and $G$ values of $(0.68,0.05)$ compared to the values $(0.90,0.00)$ for its native state (Fig. $4 \mathrm{~h}$ and Supplementary Fig. 7). All of the topological entanglements that we observe had a noncovalent lasso topology $30,31,32,33,34$, where native contacts within a certain folded region established a closed loop along the protein backbone and another segment in the same chain threaded through this loop to become entangled (Fig. 5). None of them are topologically knotted, as identified by a knot detection algorithm ${ }^{35}$, meaning that pulling on their termini will result in a fully extended conformation. Thus, all three enzymes sampled near-native, entangled but unknotted structures during folding.

Some entangled structures are long-lived kinetic traps. To disentangle a misfolded structure, it is often necessary to unfold some portion of the properly folded segments to attain the native state. This can be energetically costly. Therefore, we hypothesized that these near-native, entangled structures are long-lived kinetic traps. To test this hypothesis, we calculated the posttranslational probability of being in each metastable state as a function of time using a master equation model (Supplementary Methods Section 14.1, Supplementary equation (17)) and report the results in panels $c$ of Figs. 2, 3 and 4. For CAT-III and DDLB, entangled states (P10, P12, and $P 13)$ and (P5, P9, and P10), respectively, persisted with appreciable populations $(>0.1 \%)$ until 60 $s$ after nascent protein release from the ribosome. For DHFR, the single entangled state (P3), populated only $0.2 \%$ of the time, disentangled by $60 \mathrm{~s}$. As a further test, we examined whether any post-translational trajectory of CAT-III or DDLB that reaches an entangled state ever converts to an unentangled state. For CAT-III and DDLB, we find that $83.2 \%$ and $96.6 \%$ of trajectories sample an entangled state, and of these, $87.8 \%$ and $34.1 \%$ do not convert to a state that is not entangled by the end of the simulations. Thus, we conclude that many of these entangled structures represent long-lived kinetic traps that convert to the native state over very long time scales. 
Entangled structures have altered catalytic properties. The noncovalent lasso entanglement intermediates we observe are a form of misfolding, as they represent ordered structures that are not native. Therefore, we hypothesized that some of these entangled structures have catalytic properties different than those of the native ensemble. To test this hypothesis, we calculated the transition state barrier height of the enzyme reaction of each metastable state using a backmapping procedure to an all-atom representation and subsequent QM/MM umbrella sampling simulations of the catalytic reaction (see the potential of mean force plots in Supplementary Fig. 8-10). Thus, for each metastable state, we obtained the activation free energy barrier $\Delta G^{\ddagger}$ going from reactants to products. For all three proteins, the native state had the lowest activation energy (panel $\mathrm{f}$ in Figs. 2, 3 and 4), and the other metastable states had higher barriers. Thus, these misfolded and entangled intermediates contribute to changes in specific activity. Coupled with the observation that entangled structures tend to be long-lived kinetic traps, we also conclude that these specific metastable states lead to reduced specific activity over long time scales.

Distinctions between shallow and deep entanglements explain why CAT-III and DDLB cannot quickly fold but DHFR can. Not all entangled structures are long-lived kinetic traps; otherwise, the entangled structure of DHFR (state P3 in Fig. 4h and Supplementary Fig. 7) would never disentangle. Sliding a small number of residues out of the closed loop (Fig. 5, also see Supplementary Methods Section 11) tends to be easier than sliding a large number of residues. In addition, unfolding a small number of residues during disentanglement also tends to be easier than unfolding a large number of residues. Therefore, we hypothesized that the minimum number of residues involved in the threading segment, whose reptation can cause disentanglement, and the minimum number of residues needed to unfold during the disentanglement process should correlate with the ability of entangled metastable states to interconvert to unentangled states. To test this hypothesis, we analyzed the representative structures from each metastable state. The entanglement of DHFR involved only, on average, 5 residues in the threading segment, and sliding these segments through the loop should not cause any portion of the protein to unfold. For CAT-III and DDLB, entangled states that never disentangle in the simulations involved, on average, 34 and 8 residues, respectively, in the threading segment and had 27 and 53 residues that need to unfold during disentanglement (see https://yuj179.github.io/topo entanglements/). These results are consistent with our hypothesis and suggest that because DHFR's entanglement is 'shallow' (i.e., involves a few residues and does not need to unfold to disentangle), thermal fluctuations can easily disentangle this structure, while thermal energy is not sufficient to quickly disentangle the CAT-III and DDLB 'deep' entanglements.

Native-like entangled states are much less likely than other misfolded states to interact with chaperones, aggregate or be degraded and hence remain soluble. The proteostasis machinery in cells has the potential to catalyze the folding or degradation of these entangled structures. The entangled structures could also potentially aggregate and be removed from the pool of soluble enzymes. We accounted for the effects of these processes by estimating the aggregation, degradation and Hsp70 binding propensities of the different metastable states (see Supplementary Methods Section 14.2, Supplementary equation (21)). We observed, as expected, that the less structured metastable states have a higher propensity to aggregate, be degraded or interact with chaperones. For example, relative to the native state, states P1 through P8 of CATIII (see structures in Supplementary Fig. 5) are much more likely to experience one of these processes, as indicated by the larger magnitude of the bar plots in Fig. 2d. However, states P9 to P13 exhibit a similar propensity as the native state (P14) to aggregate, be degraded, or become chaperone substrates. Similar results are observed for DDLB (Fig. 3d) and DHFR (Fig. 4d). Thus, our model indicates that some of these entangled structures do not interact with the proteostasis machinery any more than the native state does. Therefore, the altered specific activity we observe is likely to persist inside cells over long-time scales. 
We estimated the percent of proteins in each metastable state that are likely to remain soluble by accounting for the aggregation, degradation and Hsp70 binding propensities within each metastable state (see Supplementary Methods Section 14.2, Supplementary equation (19)). We find that for both CAT-III and DDLB, many of the near-native entangled structures remained soluble. At least $94 \%$ of the proteins in entangled states P10, P12 and P13 for CAT-III are estimated to remain soluble (Fig. 2 e), while at least $89 \%$ of the proteins in entangled states P5, P9 and P10 of DDLB are expected to remain soluble (Fig. 3e). Thus, many of the long-lived, nearnative entangled states are likely to remain soluble and free from aggregation, degradation, and catalyzed folding by chaperones.

The reason for this is that these near-native structures sequester the residues and sequence motifs that promote these processes to an extent similar to that in the native state. For example, state P12 of CAT-III exposes a similar amount of hydrophobic surface area as the native state ensemble $\left(36.2 \mathrm{~nm}^{2}\right.$ versus $\left.33.9 \mathrm{~nm}^{2}\right)$. The exposed surface areas of residues that promote aggregation and interactions with the chaperone Hsp70 are also similar between state P12 and the native state, respectively, $20.4 \mathrm{~nm}^{2}$ versus $17.8 \mathrm{~nm}^{2}$ and $39.4 \mathrm{~nm}^{2}$ versus $37.3 \mathrm{~nm}^{2}$.

Entanglements persist in all-atom models. To test that the entangled structures we observe are also kinetic traps in all-atom models, we back-mapped two entangled conformations (states P12 and P13) of CAT-III and two from DDLB (states P5 and P10) to an all-atom representation (see Supplementary Methods Section 13). We then simulated three independent trajectories of each conformation in fully solvated, unrestrained molecular dynamics at $310 \mathrm{~K}$ (Supplementary Methods Section 14.4). We find that all entanglements persist in each $1 \mu \mathrm{s}$ trajectory (Supplementary Fig. 11). These results indicate that the entangled structures we have identified are likely to be long-lived kinetic traps regardless of the model resolution.

Synonymous mutations alter the post-translational populations of entangled states. Next, we examined how translation speed changes affect the post-translational populations of entangled structures. Using the master equation model, we observe that for CAT-III, $60 \mathrm{~s}$ after translation termination, $74.1 \%(95 \% \mathrm{Cl}:[71.3 \%, 76.9 \%])$ of the structures are entangled when synthesis was fast, while $71.2 \%(95 \% \mathrm{Cl}$ : $[68.1 \%, 74.0 \%])$ are entangled when synthesis was slow. Likewise, the entangled population of DDLB accounted for 34.6\% (95\% Cl: [31.64\%, 37.6\%]) and $29.4 \%(95 \% \mathrm{Cl}:[26.6 \%, 32.3 \%])$ of the total under slow and fast translation, respectively. In contrast, the population of entangled structures for DHFR remained zero regardless of translation speed $60 \mathrm{~s}$ post termination. Thus, synonymous mutations can alter the population distributions of entangled states over long-time scales for deep entanglements. 


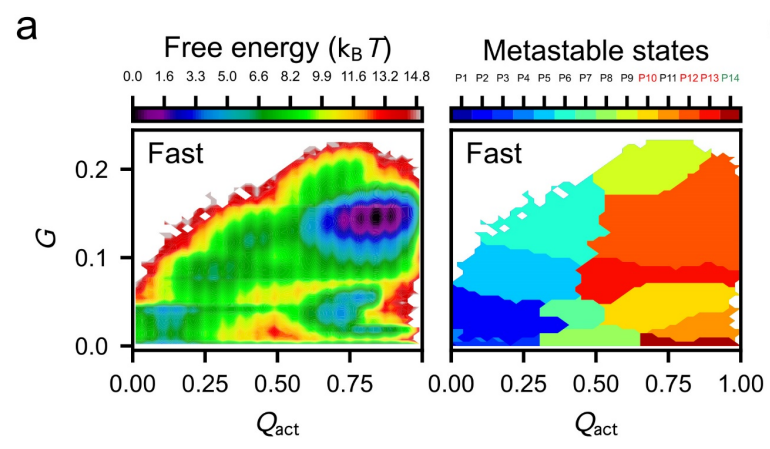

b
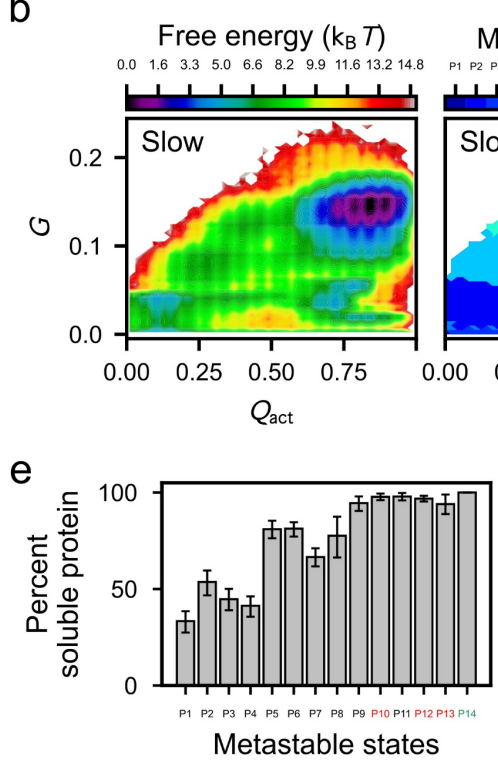

h

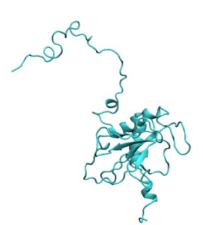

State P8

$(0.52,0.01)$

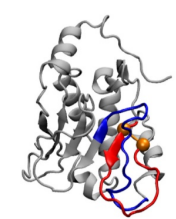

State P10

$(0.64,0.05)$
Metastable states

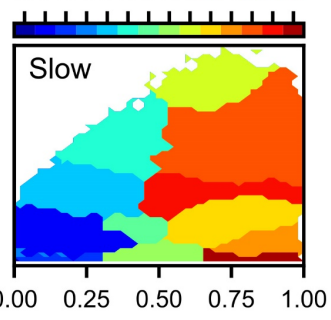

f

C
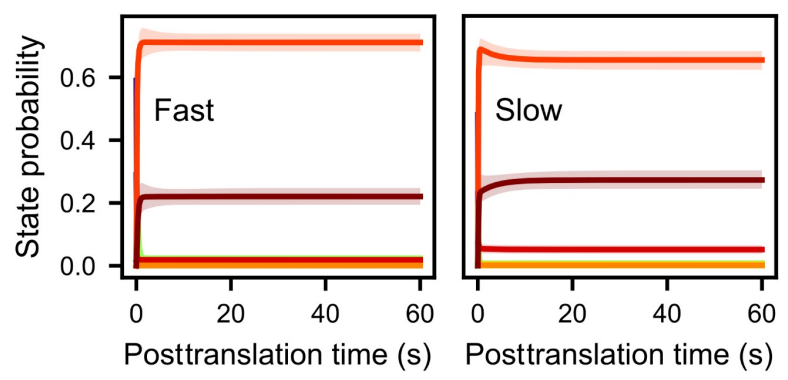

d
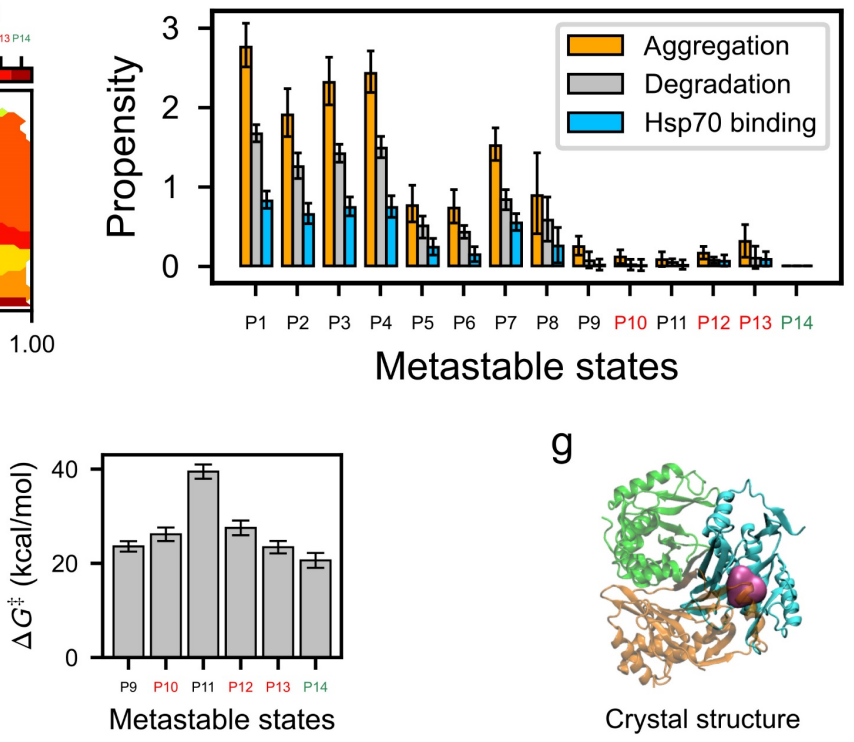

g

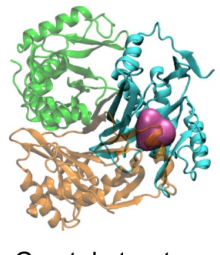

Crystal structure

Figure 2. Fast translation partitions more CAT-III into post-translational kinetically trapped entangled states. $a$ and $b$, Free energy surfaces for the post-translational folding of fast-translating (a) and slow-translating (b) mRNA variants as a function of the order parameters $Q_{\text {act }}$ and $G$ (left) and the regions corresponding to different metastable states (right). c, Time courses of gross state probabilities (soluble + insoluble; same colors as the metastable states in panels $a$ and b) with error bars shown as transparent stripes for fast (left) and slow (right) variants. $d$, The aggregation, degradation and Hsp70 binding propensities of each metastable state calculated using Supplementary equation (21). e, The percent soluble protein of each metastable state calculated by Supplementary equation (19). $\mathrm{f}$, The median transition state barrier heights $\left(\Delta G^{\ddagger}\right)$ for the near-native metastable states calculated from the QM/MM simulations. $g$, The trimer crystal structure (3cla) with substrate shown in magenta and three monomers shown in green, orange, and cyan. h, From left to right, the representative structures of the intermediate state P8; near-native kinetically trapped states P10, P12 and P13 (the closed loop and threading segment of their entangled regions are colored in red and blue, respectively); and the native state $\mathrm{P} 14$. The average Qact and $G$ coordinates for each state are reported below the structure in the format ( $Q$ act value, $G$ value). For panels a through $\mathrm{h}$, kinetically trapped entangled states are labeled in red; the native state (i.e., state P14) is labeled in green; and others are labeled in black. All error bars represent $95 \% \mathrm{Cls}$ calculated by 
bootstrapping. The structures of P10, P12 and P13 can be explored interactively at https://yuj179.github.io/topo entanglements/.

a

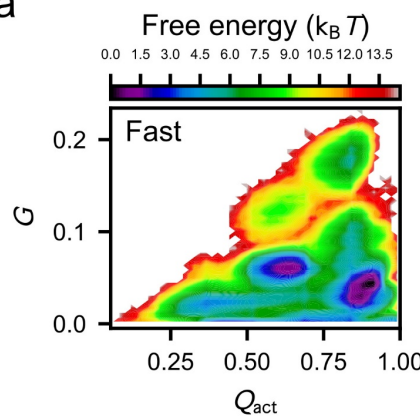

b

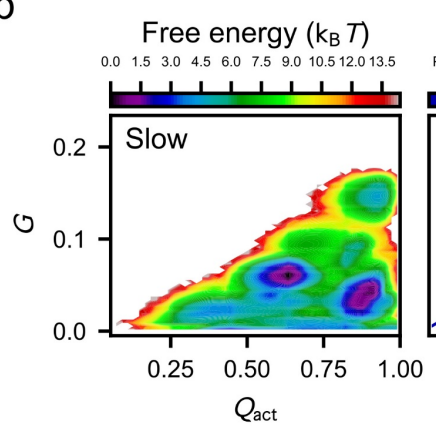

e

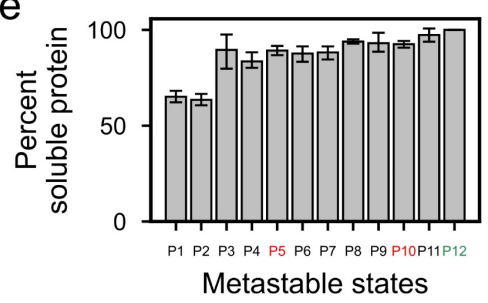

h
Metastable states P1 P2 P3 P4 P5 P6 P7 P8 P9P10P11P12

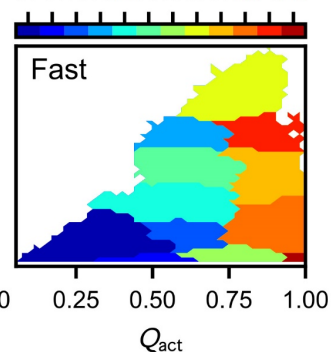

C
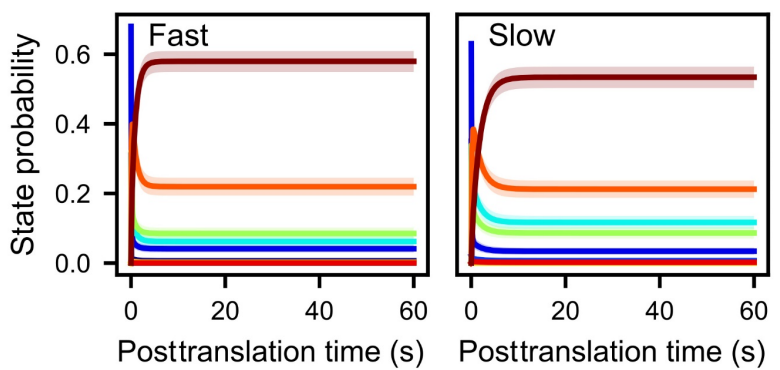

d

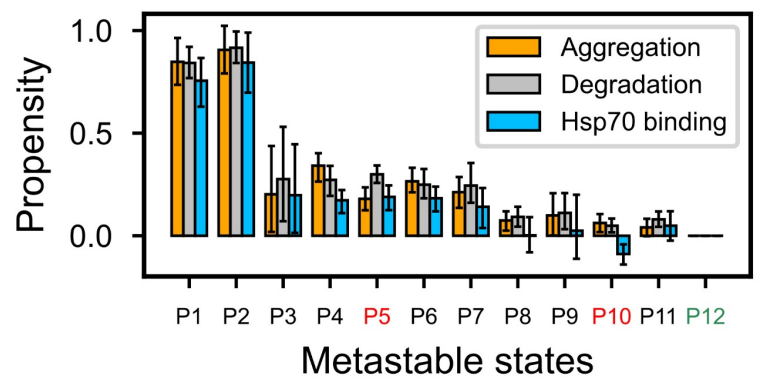

Metastable states P1 P2 P3 P4 P5 P6 P7 P8 P9 P10P11P12

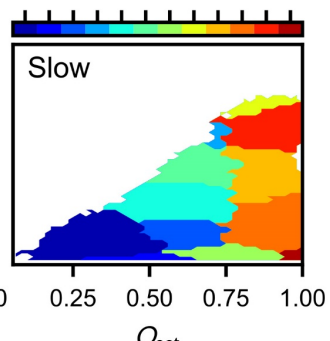

Metastable states

f
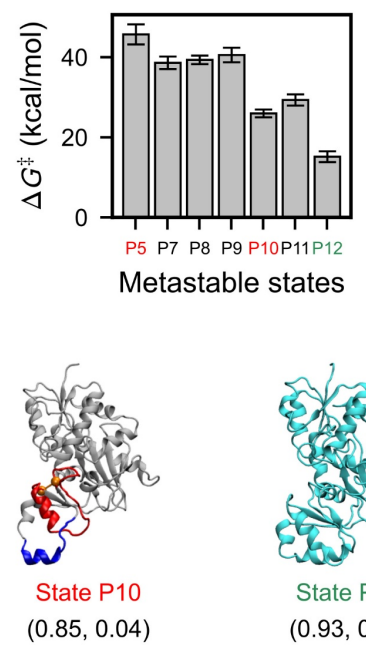

$(0.85,0.04)$ g

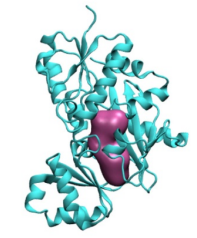

Crystal structure
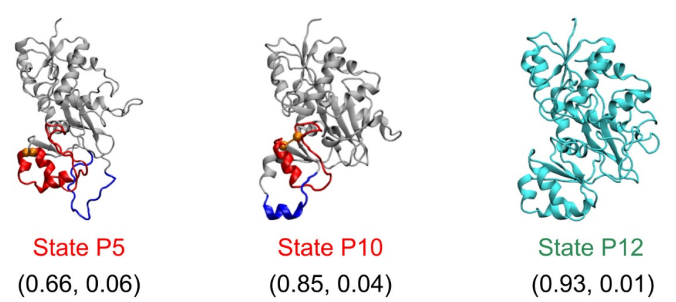

Figure 3. Slow translation partitions more DDLB into post-translational kinetically trapped entangled states. $a$ and $b$, Free energy surfaces for the post-translational folding of fast-translating (a) and slow-translating (b) mRNA variants as a function of the order parameters Qact and $G$ (left) and the regions corresponding to metastable states (right). c, Time courses of gross state probabilities (soluble + insoluble; same colors as the metastable states in panels $a$ and $b$ ) with error bars shown as transparent stripes for fast (left) and slow (right) variants. $d$, The aggregation, degradation and Hsp70 binding propensities of each metastable state calculated using Supplementary equation (21). e, The percent soluble protein of each metastable state calculated by Supplementary equation (19). f, The median transition state barrier heights $\left(\Delta G^{\ddagger}\right)$ for the near-native metastable states calculated from the QM/MM simulations. State P4 and P6 are excluded from the $\Delta G^{\ddagger}$ estimation due to the low probability of being populated in the simulations $(<0.05 \%)$. $\mathrm{g}$, Crystal structure (4c5c) with substrate shown in magenta. $\mathrm{h}$, from left to right, the representative structures of the near-native kinetically trapped states P5 and P10 (the closed loop and threading segment of their entangled regions are colored in red and blue, respectively) and the native state P12. The average 
Qact and $G$ coordinates for each state are reported below the structure in the format (Qact value, $G$ value). For panels a through $\mathrm{h}$, kinetically trapped entangled states are labeled in red; the native state (i.e., state $\mathrm{P} 12$ ) is labeled in green; and others are labeled in black. All error bars represent $95 \% \mathrm{Cls}$ calculated by bootstrapping. The structures of P5 and P10 can be explored interactively at https://yuj179. github.io/topo entanglements/.

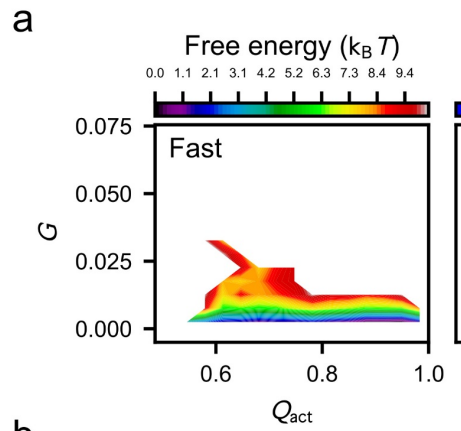

b

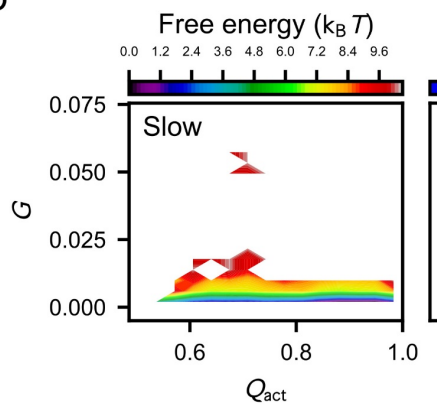

e

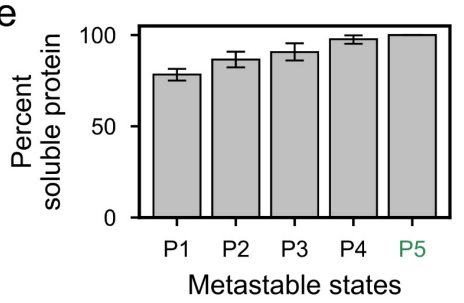

Metastable states
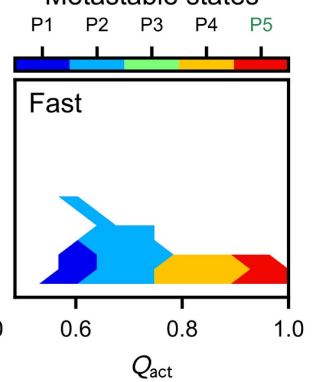

Metastable states
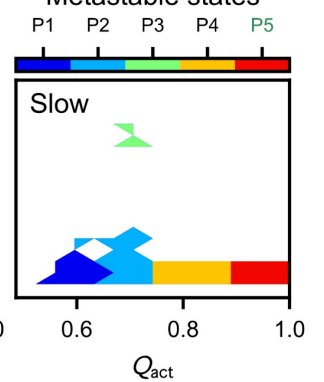

f

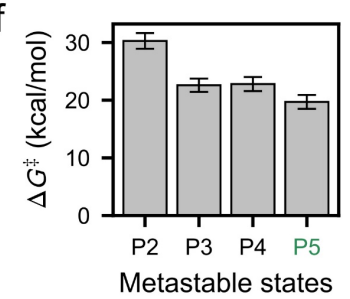

$\mathrm{C}$
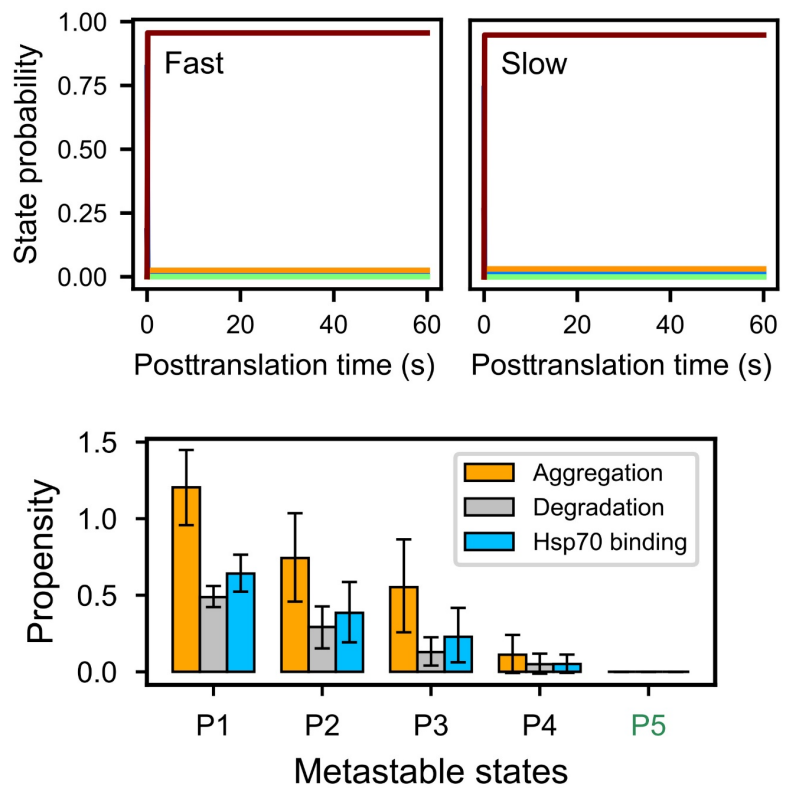

g

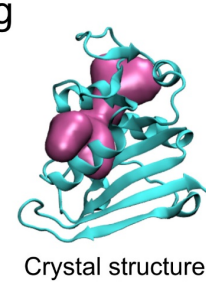

h

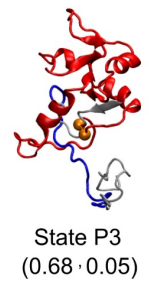

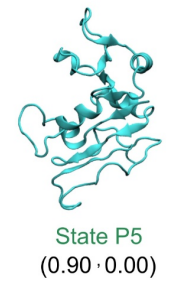

Figure 4. No kinetically trapped states arise in synonymous variants of DHFR. $a$ and $b$, Free energy surfaces for the post-translational folding of fast (a) and slow (b) mRNA variants as a function of the order parameters Qact and $G$ (left) and the regions corresponding to metastable states (right). c, Time courses of gross state probabilities (soluble + insoluble; same colors as the metastable states in panels a and b) with error bars shown as transparent stripes for fast (left) and slow (right) variants. $d$, The aggregation, degradation and Hsp70 binding propensities of each metastable state calculated using Supplementary equation (21). e, The percent soluble protein of each metastable state calculated by Supplementary equation (19). $f$, The median transition state barrier heights $\left(\Delta G^{\ddagger}\right)$ for the near-native metastable states calculated from the QM/MM simulations. g, Crystal structure (4kjk) with substrate shown in magenta. $h$, From left to right, the representative structures of transient misfolded state P3 (the closed loop and threading segment of the shallow entangled regions are colored in red and blue, respectively) and native state P5. The average $Q_{\text {act }}$ and $G$ coordinates for each state are reported below the structure in the format ( $Q$ act value, $G$ value). For panels a through $\mathrm{h}$, kinetically trapped entangled states are labeled in red, the native state (i.e., state P5) is labeled in green, and others are labeled in black. All error bars represent $95 \%$ Cls calculated by bootstrapping. The structure of P3 can be explored interactively at https://yuj179. github.io/topo entanglements/. 


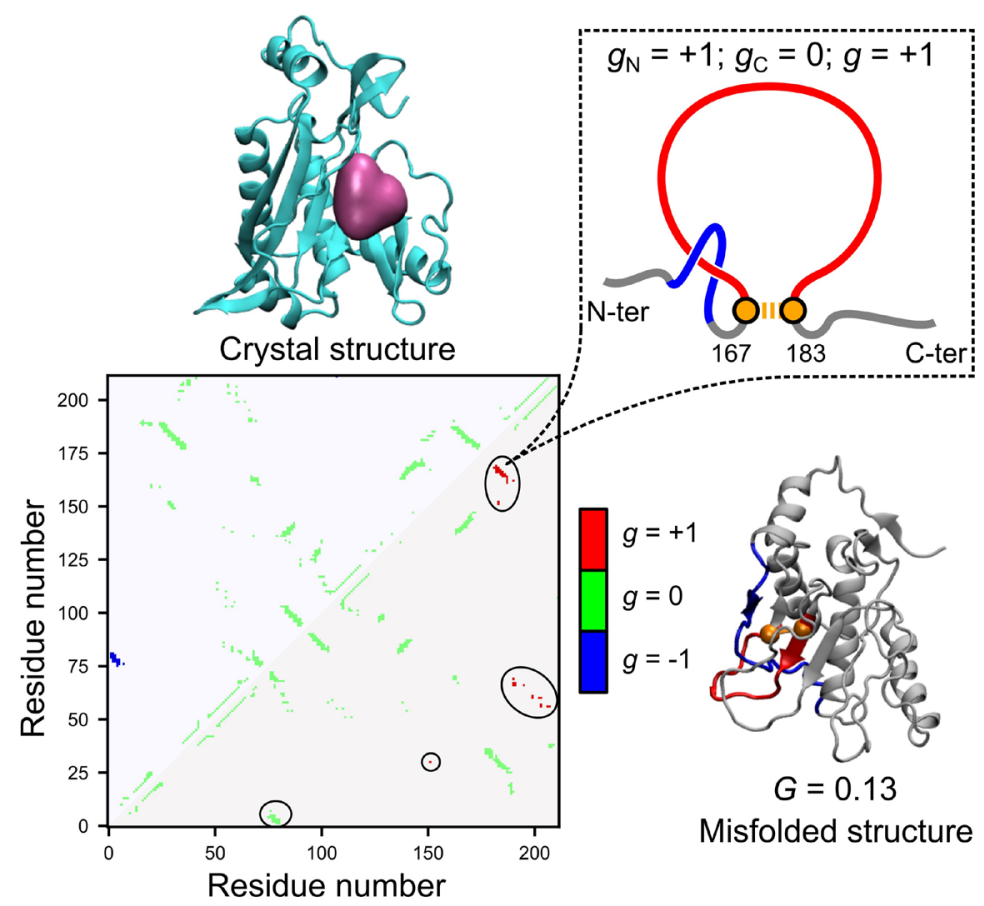

Figure 5. Illustration of the $G$ metric and the noncovalent lasso topology. A contact map of the native contacts is presented on the bottom left, where the upper and lower triangular regions represent the native contacts within the native crystal structure and the misfolded structure, respectively. The native contacts are colored based on the linking number $g$ calculated by Supplementary equation (16). A topology diagram of the noncovalent lasso entanglement formed by the native contact of residues 167 and 183, as an instance, is presented at the top right, where the closed loop is shown in red and the threading segment is shown in blue. As described by equation (2), the $G$ metric of this misfolded structure, $G=0.13$, was estimated by counting the number of native contacts within the misfolded structure, whose $g$ value is different from that of the same native contact within the crystal structure (i.e., the number of native contacts covered by the black circles on the lower triangular region of the contact map), and normalizing them to the total number of native contacts in the crystal structure.

Synonymous mutations cause a divergence in the distribution of co-translational structures that persist post-translationally. The post-translational changes in the distribution of entangled structures must have originated co-translationally. We hypothesized that the changes in translation speed alter the co-translational folding pathways of the protein. To test this hypothesis, we first assessed how different were the nascent chain structural distributions under fast and slow translation. To do this, we applied the normalized Jensen-Shannon divergence metric (Supplementary equation (23)) to the population of microstates identified as part of our Markov state modeling. A value of 0 means there is no difference between the distributions, while a value of 1 means the distributions are completely different. Calculating this metric at each nascent chain length during synthesis, we found that for all three proteins, the structural divergence induced by synonymous mutations was small at short nascent chain lengths and tended to increase as the nascent chain length increased (Fig. 6b, e and h). The maximum divergence occurred at or near the longest nascent chain length before the nascent chain was released from the ribosome. Specifically, for DHFR, the structural ensembles arising from fast and slow synthesis started to diverge at approximately 110 residues in length, while for CAT-III and DDLB, the two distributions start to diverge at 190 and 210 residues, respectively. Thus, the fast- and slow-translation rates altered the co-translational distributions of conformations for all three enzymes. 
Applying this divergence metric to the time-dependent post-translational structural distributions, we observed that for DHFR, the initially different structural ensembles arising from fast and slow synthesis rapidly converged to nearly the same distribution post-translationally (i.e., have divergence values very close to zero in Fig. 6h). For CAT-III and DDLB, the two distributions do not reconverge $60 \mathrm{~s}$ after synthesis. Thus, the changes in conformations caused by synonymous mutations are quickly 'forgotten' by DHFR due to its rapid equilibration, while CAT-III and DDLB retain a 'memory' of those co-translational differences due to kinetic trapping.

Changes in co-translational folding pathways give rise to altered post-translational populations. Next, we characterized the co- and post-translational folding pathways arising from changes in the translation rate. To do this, we calculated the populations and pathway probabilities of transitions between metastable states occurring co- and post-translationally under the different translation schedules (see Supplementary Methods Section 16). To display similarities and differences in folding pathways, we used arrows with different colors. Black arrows between metastable states indicate that a transition was observed between those states in the top $80 \%$ of populated pathways, blue arrows indicate that the transition was seen only in the slowtranslation schedule, and orange arrows indicate that the transition was seen only in the fast translation schedule.

We identified nine co- and five post-translational metastable states (representative structures shown in Fig. 6g) for DHFR. The co- and post-translational folding pathways were very similar, as most of the transitions were seen in both translation schedules (black arrows in Fig. $6 \mathrm{~g}$ ). There were some differences though. Transitions from state $\mathrm{C} 7$ to $\mathrm{C} 9$ and from $\mathrm{C} 9$ to P1 were observed for only fast and slow schedules, respectively. The pathway probabilities, however, demonstrated that even though the initial post-translational structural distribution differed, nearly all states converted to the native state by the end of the post-translational simulations (Fig. 6i). For example, the pathway probabilities of $\mathrm{C} 9 \rightarrow \mathrm{P} 5$ are $100 \%$ for both fast and slow translation. The pathway probability of C8 $\rightarrow$ P5 is $100 \%$ for fast translation and $99.8 \%$ for slow translation. An exception is the transition probability of $\mathrm{C} 6 \rightarrow \mathrm{P} 5$, where state $\mathrm{C} 6$ was not sampled by the slow variant, and no such transition was observed in our simulation. However, due to the small population of C6 (2\% in Fig. 6i) sampled by the fast variant, this difference was negligible. Thus, the co-translational folding of DHFR was slightly affected by differences in translation speed; however, these differences quickly disappeared due to rapid folding from all post-translational metastable states.

In contrast, CAT-III exhibited a co- and post-translational folding reaction network (Fig. 6a) and pathway probabilities that were sensitive to translation speed changes and whose resulting differences persisted post-translationally. Nine co-translational and fourteen post-translational metastable states were identified for CAT-III (Fig. 6a). Six of these post-translational metastable states (P6, P9, P10, P11, P12 and P13) exhibited entangled structures, while no co-translational states exhibited entanglement. Thus, entanglement was a post-translational process for CAT-III (see Supplementary videos I and II visualizing, respectively, the process of entanglement versus correct folding). Very different co-translational folding pathways were observed in the top $80 \%$ of pathways starting at a nascent chain length of approximately 195 residues, where the divergence metric exhibited a large increase (Fig. 6b), and transitions into and out of co-translational metastable state C9 started to differ between the fast and slow translation schedules (as indicated by the blue and orange arrows in Fig. 6a). For example, only in the slow schedule can C9 transition to P13, while only in the fast schedule can C5 transition to P1. These differences in allowed transitions persisted post-translationally as well, with a number of states, such as P12 and P13, being effective sinks during the post-translational simulations, i.e., allowing no direct or indirect transitions to the native state (P14) once they were reached. These sinks are entangled structures, which is consistent with our earlier observation that these entangled states tend to be kinetic traps (indicated by the red borders around metastable states P12 and P13 in Fig. 6a). 
Thus, the translation speed causes differences in the CAT-III co-translational folding pathways once at least 195 residues have been synthesized, and those differences lead to changes in the populations of post-translationally entangled states, thereby altering the transition state barrier for the reaction this enzyme carries out (Fig. $2 f$ ) and affecting the specific activity of CAT-III over long time scales (Fig. 1g).

Finer-grained consideration of the folding pathways (estimated by using the master equation model) shows that post-translationally, CAT-III fast partitioned $5.7 \%(95 \% \mathrm{Cl}[1.7 \%, 9.9 \%])$ more protein (gross amount, soluble + insoluble, same hereafter) into the near-native kinetic trap P12, while CAT-III slow partitioned $5.2 \%(95 \% \mathrm{Cl}[1.4 \%, 9.2 \%])$ more protein into native state P14. The transitions towards the native state P14 were enhanced for CAT-III slow, whereas the transitions towards the kinetic trap P12 were enhanced for CAT-III fast. This is also indicated by the pathway probabilities shown in Fig. 6c. Synonymous mutations also led to smaller changes in other states, including the kinetic trap P13 and intermediate state P8.

Similar to CAT-III, DDLB also exhibits co- and post-translational folding pathways that were sensitive to changes in translation speed across its eight co- and twelve post-translational metastable states. However, unlike CAT-III, DDLB co-translationally formed entangled structures (states C8 and C9) that persisted to form entangled post-translational states (states P1, P3 and P5). In CAT-III, the divergence in co-translational folding pathways between fast and slow translation schedules monotonically increased with increasing chain length (Fig. 6b). However, the co-translational folding pathways of DDLB diverged starting at nascent chain length 110 but reconverged around 190 residues, diverging again starting at 210 residues (Fig. 6e). The first divergence involved a transition to state $\mathrm{C} 4$ with the fast schedule that did not occur with the slow schedule (orange arrow in Fig. 6d). This state ultimately interconverted to state C3, which is an obligatory intermediate in both schedules. The second divergence started at state $\mathrm{C} 7$, in which $6 \%$ more nascent chains went to entangled state C8 with the slow schedule than with the fast schedule, and the rest went to state $\mathrm{C} 10$. Parallel co- and post-translational folding pathways then arose. In one pathway, transitions were observed from $\mathrm{C} 10 \rightarrow \mathrm{C} 11 \rightarrow \mathrm{P} 2$, while the other transitions from C8 $\rightarrow$ C9 $\rightarrow$ P1 involved entangled structures in all states (see Fig. 6d, also see Supplementary videos III and IV illustrating the processes of entanglement and folding). The increased probability of this entangled pathway during slow translation decreased the probability that DDLB would posttranslationally reach the native state P12 (3.1\% versus $9.3 \%$ in slow versus fast, Fig. 6 f), while DDLB $_{\text {slow }}$ partitioned $5.5 \%(95 \% \mathrm{Cl}[3.1 \%, 7.8 \%])$ more protein (gross amount, soluble + insoluble) into the near-native kinetic trap P5 after post-translation (as estimated by using the master equation model). Thus, we again see that entangled states act as sinks with altered transition state barriers for the enzyme's reaction (Fig. 3f). This population shifts among these states and the folding pathways they take part in leads to long-lived changes in specific activity. 
a

b

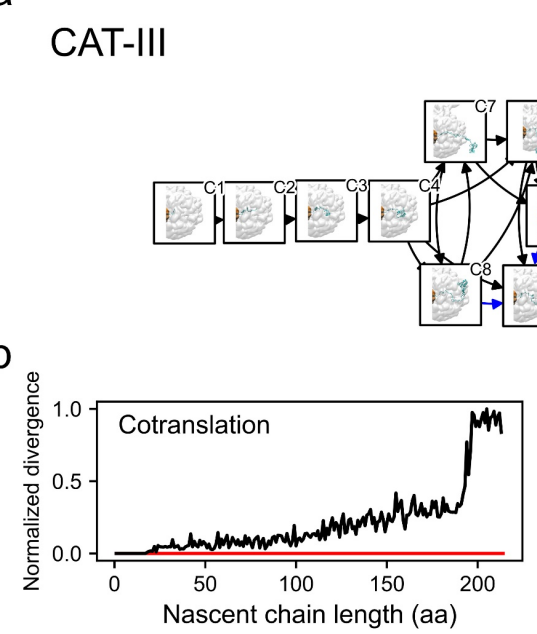

d

e

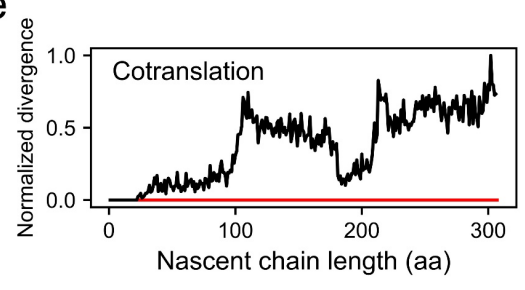

DDLB

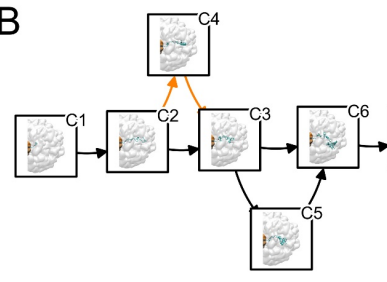

Nascent chain length (aa)

\section{g}

DHFR

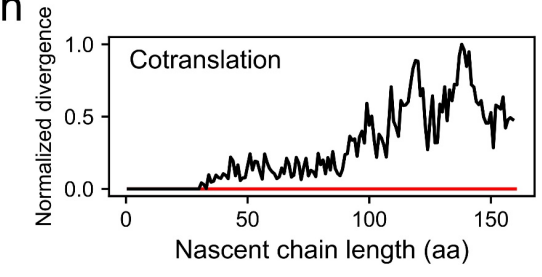

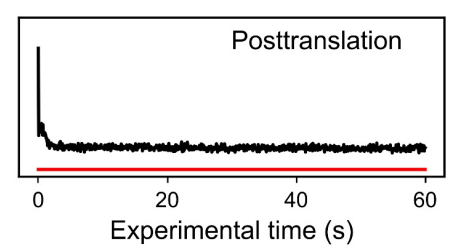

Experimental time (s)
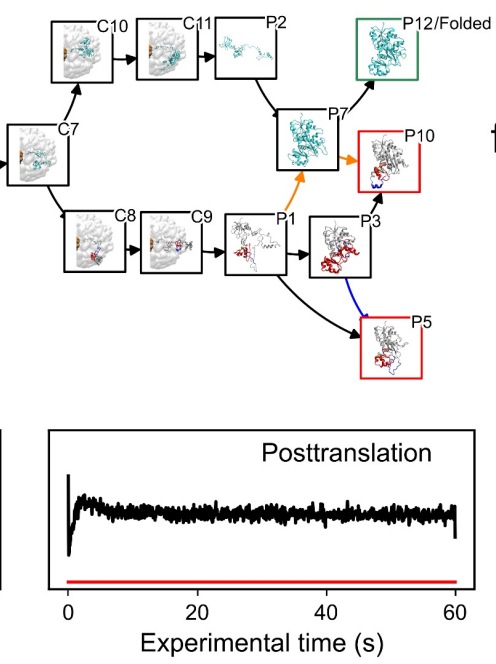

$\mathrm{C}$

\begin{tabular}{|l|c|c|}
\hline \multicolumn{3}{|c|}{ Pathway probability } \\
\hline & Fast & \multicolumn{1}{|c|}{ Slow } \\
\hline${ }^{*} \rightarrow \mathrm{C} 9$ & $7.0 \%$ & $24.0 \%$ \\
\hline $\mathrm{C} 9 \rightarrow \mathrm{P} 14 /$ Folded & $14.3 \%$ & $12.9 \%$ \\
\hline${ }^{*} \rightarrow \mathrm{C} 6$ & $63.0 \%$ & $53.0 \%$ \\
\hline $\mathrm{C} 6 \rightarrow \mathrm{P} 14 /$ Folded & $20.5 \%$ & $30.0 \%$ \\
\hline${ }^{*} \rightarrow \mathrm{C} 5$ & $30.0 \%$ & $23.0 \%$ \\
\hline $\mathrm{C} 5 \rightarrow \mathrm{P} 14 /$ Folded & $28.3 \%$ & $35.7 \%$ \\
\hline
\end{tabular}

f
\begin{tabular}{|l|r|r|}
\hline \multicolumn{3}{|c|}{ Pathway probability } \\
\hline & \multicolumn{1}{|c|}{ Fast } & \multicolumn{1}{c|}{ Slow } \\
\hline${ }^{*} \rightarrow \mathrm{C} 11$ & $70.0 \%$ & $64.0 \%$ \\
\hline $\mathrm{C} 11 \rightarrow \mathrm{P} 12 /$ Folded & $79.6 \%$ & $81.1 \%$ \\
\hline${ }^{*} \rightarrow \mathrm{C} 9$ & $30.0 \%$ & $36.0 \%$ \\
\hline $\mathrm{C} 9 \rightarrow \mathrm{P} 12 /$ Folded & $9.3 \%$ & $3.1 \%$ \\
\hline
\end{tabular}

Figure 6. Co- and post-translational folding pathways of CAT-III, DDLB and DHFR. a, d, and g, The most probable folding pathways are presented as a directed graph where the nodes represent metastable states, with representative structures shown in the boxes, and the edges represent the transitions in the $80 \%$ most likely pathways. The nodes corresponding to the native state and the kinetically trapped states 
are framed in green and red, respectively. The transitions (edges) that are observed in only the fast variants or the slow variants are marked in orange and blue, respectively. b, e and h, Normalized Jensen-Shannon divergence (Supplementary equation (23)) of the co- and post-translational microstate distributions comparing fast and slow variants, with a red line indicating zero divergence. $\mathrm{c}$, $\mathrm{f}$ and $\mathrm{i}$, The co-translational pathway probabilities $\left(^{*} \rightarrow\right.$ end state $C^{*}$ ) and the post-translational pathway probabilities from the cotranslational end states to the native state $\left(\mathrm{C}^{*} \rightarrow\right.$ native state) are summarized in the tables. The pathway probabilities whose changes were greater than $5 \%$ are highlighted in yellow. The post-translational pathway probabilities are normalized by the corresponding co-translational pathway probabilities that involve the particular co-translational end state.

\section{Discussion}

How synonymous mutations alter the specific activity of enzymes has been an unanswered question. A detailed understanding of the molecular mechanisms giving rise to this phenomenon would provide biochemists, molecular biologists, evolutionary biologists, and biomedical researchers a framework to interpret experiments on the influence of codon usage on protein structure, function, cellular phenotype, disease, and the selection pressures shaping mRNA sequence evolution. In this study, we developed a novel multiscale model that recapitulates the experimental observations for the specific activity of CAT-III variants ${ }^{1}$ and correctly predicts the changes in specific activity of DDLB variants, giving us confidence that the model is realistic. This provided us the opportunity to study the structures, pathways, and kinetics that give rise to this phenomenon.

Our key findings are that (1) changes in elongation kinetics induced by synonymous mutations can alter co-translational nascent chain structural ensembles and folding pathways; (2) for some enzymes, such as CAT-III and DDLB, these changes in the structural ensemble can persist long after the nascent chain has been released from the ribosome; (3) this persistence arises from conformational states that are long-lived kinetic traps; (4) these kinetic traps arise at the molecular level from deep entanglements that slowly disentangle because they require the unfolding of already folded protein segments; (5) these entanglements are non-covalent lasso topologies in which a closed loop is formed by a backbone segment connecting two residues that form a noncovalent native contact, and another segment threads through this loop; (6) many of these entangled structures have decreased catalytic efficiencies due to structural perturbation of their active sites; (7) some entangled structures are very similar to the native state - exposing similar extents of hydrophobic surface area and chaperone binding motifs; and (8) because of their nearnative conformations, these entangled structures can bypass the chaperone and degradation machinery of the cell and do not exhibit an increased propensity to aggregate. This situation results in a soluble fraction of enzymes with decreased specific activities that can persist for long time periods in cells.

Two concepts central to our explanation - intra-molecular entanglement and subpopulations of kinetically trapped states - are not without precedent. The material properties of entangled synthetic polymers have long been studied and modeled ${ }^{36,37}$ in the field of polymer physics. And there has been a large amount of research focused on knotted proteins that often contain disulfide bonds or topologies in which when the protein's ends are pulled in opposite directions the protein does not fully extend ${ }^{31}, 38,39,40$. These characteristics, however, are not present in our entanglements. The entanglements we observe form a closed loop due to a non-covalent native contact, and if both ends of the protein were pulled disentanglement would occur. Recently, this type of entanglement has been detected in almost one third of the protein structures deposited in the Protein Data Bank ${ }^{32}$. Thus, the potential for protein segments to form non-native, noncovalent lassos is plausible. Kinetically trapped states of proteins have been observed in single-molecule 
experiments probing the functioning of flavoenzymes ${ }^{41}, 42$. In one study, heterogeneous populations of the protein cholesterol oxidase were observed to stochastically switch very slowly between active and nonactive states ${ }^{41}$. However, in that study, the influence of protein synthesis on the distribution of conformational states was not probed. Thus, a novel aspect of our discovery is that it combines the phenomena of entanglement and kinetic trapping as essential to the mechanism by which synonymous mutations affect co- and post-translational protein structure and function.

We also observed in our simulations the counterintuitive phenomenon that the folding of some proteins is promoted when their rapid synthesis gives them less time to fold on the ribosome. This phenomenon was first predicted from chemical kinetic models ${ }^{43}$ of co-translational folding. Specifically, we observed that synthesizing DDLB three times faster increased the final posttranslational folding probability of DDLB by $4.6 \%(95 \% \mathrm{Cl}[0.4 \%, 8.9 \%])$ and increased its activity. Consistent with the earlier prediction ${ }^{43}$, this phenomenon arose because having less time to fold on the ribosome decreases DDLB partitioning into the misfolded, kinetically trapped state. This adds to the growing list of proteins for which slowing synthesis decreases folding efficiency, which results in altered function, and challenges the common rule of thumb that slow-translating codons promote folding.

An important aspect of our choice of protein systems is that we included a protein, DHFR, whose activity is not sensitive to synonymous mutations. This provided us with the opportunity to gain insights by comparing and contrasting its behavior with those of CAT-III and DDLB. A key insight from this comparison is that even though the DHFR co-translational structures and folding pathways were sensitive to synonymous mutations, these differences did not persist posttranslationally because the only entangled state that DHFR populates and disentangled rapidly. This is consistent with previous experimental and theoretical studies that show that DHFR has fast folding kinetics and an absence of significant off-pathway intermediates ${ }^{44}$ and kinetic traps ${ }^{45}$. We demonstrated that the two reasons for this are that DHFR only negligibly populates an entangled conformation and that the entanglement it forms is 'shallow' - its disentanglement requires only 5 residues to slide out of the closed loop and does not need to unfold any other portions of the protein to do so. Thus, this shallow entanglement can be easily disentangled without large structural rearrangements. In contrast, CAT-III and DDLB entanglements have many more residues involved, requiring large structural rearrangements to become disentangled, leading to more-persistent entangled structures. Thus, the presence of entanglement is not sufficient to guarantee a kinetic trap. What is important is the nature of the entanglement and the native structure surrounding it.

Like any simulation study, limitations of force fields, time scales, and sampling statistics are of concern. In the CG simulations, we used a structure-based force field to encode the native state as the global free energy minimum. One of the many benefits of this is that we automatically know that the entangled structures we observe are metastable states, as the native state is encoded to be the global free energy minimum below the protein's melting temperature. However, one potential drawback is that this force field does not allow ordered, nonnative secondary and tertiary structures to form (e.g., the model does not allow $\beta$-sheets to switch to $\alpha$-helical bundles). This means that additional structures beyond entanglement could also contribute to the influence of synonymous mutations on enzyme activity. At the monomeric protein level, however, such nonnative ordered structure seems unlikely. Except for fold-switching proteins, which are rare in nature (only 0.5 to $4 \%$ of proteins in the Protein Data Bank are estimated to switch folds ${ }^{46}$ ) and usually require quaternary interactions to induce structural changes ${ }^{47}$, there have been no reported experimental data for alternative, ordered tertiary structures occurring on the folding pathways of monomeric proteins in bulk solution. In the QM/MM simulations, we used the 3rdorder density-functional tight-binding (DFTB) method $^{48}$ as a balance between computational 
speed and accuracy. Transition state barrier heights can change depending on the level of theory and basis set used. However, it is often the case that trends across related compounds or conformations are robust. Therefore, the differences in the barrier heights between metastable states are likely to be qualitatively correct, meaning our overall conclusions would remain unchanged even if a higher level of theory was used.

Another limitation of our simulations is that we only simulated one protein chain at a time. Thus, we can never observe homodimer-swapped structures that could also be long-lived kinetic traps and influence function ${ }^{49}$. However, as noted, in the experimental examples provided in the Introduction, such dimer-swapped structures were ruled out. To address sampling issues, we applied rigorous statistical analyses of all our reported quantities, including confidence intervals and $p$-values. All our conclusions were drawn from statistically significant signals, indicating that sampling was not an issue. For these reasons, our results are robust and statistically meaningful, giving us confidence that they represent realistic scenarios of what is happening at the molecular level.

The predictions that monomeric enzymes can become intramolecularly entangled and populate long-lived states can be tested experimentally. Ensemble-level experiments, such as NMR and X-ray crystallography, often need appreciable populations of a state to detect that state, which might make entanglement detection difficult. Single-molecule techniques, such as FRET, can detect heterogeneous populations but contain little structural information since the signal arises from only the two points where dyes are attached to a protein. Therefore, cryo-EM, with its ability to build structural clusters of heterogeneous populations, and chemical-exchange mass spectrometry, which can detect time-dependent distributions of chemical modifications on proteins, seem the most promising techniques to experimentally detect these entanglements.

This study explains how synonymous mutations can alter enzyme activity in cells. Synonymous mutations alter translation elongation speeds and change the population of nascent chain conformations in entangled states that are near native but have lower catalytic efficiencies than that of the native state. Hence, the specific activity, a quantity averaged over the populations of proteins in different conformational states, can increase or decrease due to synonymous mutations. The experimental search for these entangled structures and their roles in influencing protein structure, function, and phenotypes in cells is likely to be a fruitful area of research in the future. 


\section{Acknowledgements}

E.P.O. acknowledges support from the National Science Foundation (MCB-1553291) as well as the National Institutes of Health (R35-GM124818). S.J.B. acknowledges support from the NIH (GM-122595), the Eberly Family Distinguished Chair in Science, and the Howard Hughes Medical Institute. Computations in this work have been carried out on the Extreme Science and Engineering Discovery Environment (XSEDE) supercomputer, which is supported by MCB160069, and the Pennsylvania State University's Institute for Computational and Data Sciences' Roar supercomputer.

\section{Author contributions}

E.P.O. designed the research; Y.J. developed the computational methods with contributions from E.P.O.; Y.J. wrote the computer code and carried out the simulations and computations; S.S.N. and S.J.B. designed the experimental validation for DDLB variants; S.S.N. and P.P. performed the experiments; All of the authors analyzed the data and wrote the manuscript.

\section{Code availability}

All computer code developed in this work is available in the GitLab repositories https://git.psu.edu/yuj179/cg simtk protain folding and https://git.psu.edu/yuj179/activationenergy-estimation-workflow, under the MIT license. Detailed instructions of code usage, basic theory and examples of the input/output are available in the wiki pages of the above repositories.

\section{Data and biological materials availability}

Raw data for Figures 1 to 6 are available in the supplementary data. The input data that we used to perform the simulations in this study are available in the repository subdirectory https://git.psu.edu/yuj179/cg simtk protain folding/-/blob/master/example/input data.tar.bz2. All the data that support the findings of this study, as well as the biological materials that were used for testing the enzymatic activity of DDLB variants, are available from the corresponding author upon reasonable request. 


\section{References}

1. Komar AA, Lesnik T, Reiss C. Synonymous codon substitutions affect ribosome traffic and protein folding during in vitro translation. FEBS Lett 1999, 462(3): 387-391.

2. Zhao F, Yu C-h, Liu Y. Codon usage regulates protein structure and function by affecting translation elongation speed in Drosophila cells. Nucleic Acids Res 2017, 45(14): 84848492.

3. Spencer PS, Siller E, Anderson JF, Barral JM. Silent substitutions predictably alter translation elongation rates and protein folding efficiencies. J Mol Biol 2012, 422(3): 328335.

4. Hunt R, Hettiarachchi G, Katneni U, Hernandez N, Holcomb D, Kames J, et al. A single synonymous variant (c. 354G> A [p. P118P]) in ADAMTS13 confers enhanced specific activity. International journal of molecular sciences 2019, 20(22): 5734.

5. Crombie T, Boyle JP, Coggins JR, Brown AJ. The folding of the bifunctional TRP3 protein in yeast is influenced by a translational pause which lies in a region of structural divergence with Escherichia coli indoleglycerol-phosphate synthase. Eur J Biochem 1994, 226(2): 657-664.

6. Walsh IM. Testing the Effects of Synonymous Codon Usage on Co-Translational Protein Folding Using Novel Experimental and Computational Techniques. University Of Notre Dame, 2019.

7. Yu C-H, Dang Y, Zhou Z, Wu C, Zhao F, Sachs MS, et al. Codon usage influences the local rate of translation elongation to regulate co-translational protein folding. $\mathrm{Mol} \mathrm{Cell}$ 2015, 59(5): 744-754.

8. Walsh IM, Bowman MA, Santarriaga IFS, Rodriguez A, Clark PL. Synonymous codon substitutions perturb cotranslational protein folding in vivo and impair cell fitness. Proc Natl Acad Sci 2020, 117(7): 3528-3534.

9. Sala AJ, Bott LC, Morimoto RI. Shaping proteostasis at the cellular, tissue, and organismal level. J Cell Biol 2017, 216(5): 1231-1241.

10. Liu Y, Tan YL, Zhang X, Bhabha G, Ekiert DC, Genereux JC, et al. Small molecule probes to quantify the functional fraction of a specific protein in a cell with minimal folding equilibrium shifts. Proc Natl Acad Sci 2014, 111(12): 4449-4454.

11. Buhr F, Jha S, Thommen M, Mittelstaet J, Kutz F, Schwalbe H, et al. Synonymous codons direct cotranslational folding toward different protein conformations. Mol Cell 2016, 61(3): 341-351. 
12. Martelli PL, Fariselli P, Casadio R. Prediction of disulfide-bonded cysteines in proteomes with a hidden neural network. Proteomics 2004, 4(6): 1665-1671.

13. O'Brien EP, Christodoulou J, Vendruscolo M, Dobson CM. Trigger factor slows cotranslational folding through kinetic trapping while sterically protecting the nascent chain from aberrant cytosolic interactions. J Am Chem Soc 2012, 134(26): 10920-10932.

14. Sharma AK, Bukau B, O'Brien EP. Physical origins of codon positions that strongly influence cotranslational folding: A framework for controlling nascent-protein folding. J Am Chem Soc 2016, 138(4): 1180-1195.

15. Fritch B, Kosolapov A, Hudson P, Nissley DA, Woodcock HL, Deutsch C, et al. Origins of the mechanochemical coupling of peptide bond formation to protein synthesis. J Am Chem Soc 2018, 140(15): 5077-5087.

16. Nissley DA, O'Brien EP. Structural Origins of FRET-Observed Nascent Chain Compaction on the Ribosome. J Phys Chem B 2018, 122(43): 9927-9937.

17. Leininger SE, Trovato F, Nissley DA, O'Brien EP. Domain topology, stability, and translation speed determine mechanical force generation on the ribosome. Proc Natl Acad Sci 2019, 116(12): 5523-5532.

18. Nissley DA, Vu QV, Trovato F, Ahmed N, Jiang Y, Li MS, et al. Electrostatic interactions govern extreme nascent protein ejection times from ribosomes and can delay ribosome recycling. J Am Chem Soc 2020, 142(13): 6103-6110.

19. Dunkle JA, Wang L, Feldman MB, Pulk A, Chen VB, Kapral GJ, et al. Structures of the bacterial ribosome in classical and hybrid states of tRNA binding. Science 2011, 332(6032): 981-984.

20. Arenz S, Bock LV, Graf M, Innis CA, Beckmann R, Grubmüller H, et al. A combined cryoEM and molecular dynamics approach reveals the mechanism of ErmBL-mediated translation arrest. Nat Commun 2016, 7: 12026.

21. Sharma AK, Sormanni P, Ahmed N, Ciryam P, Friedrich UA, Kramer G, et al. A chemical kinetic basis for measuring translation initiation and elongation rates from ribosome profiling data. PLoS Comp Biol 2019, 15(5): e1007070.

22. Fluitt A, Pienaar E, Viljoen $\mathrm{H}$. Ribosome kinetics and aa-tRNA competition determine rate and fidelity of peptide synthesis. Comput Biol Chem 2007, 31(5-6): 335-346. 
23. Eastman P, Swails J, Chodera JD, McGibbon RT, Zhao Y, Beauchamp KA, et al. OpenMM 7: Rapid development of high performance algorithms for molecular dynamics. PLoS Comp Biol 2017, 13(7): e1005659.

24. Nagano N. EzCatDB: the enzyme catalytic-mechanism database. Nucleic Acids Res 2005, 33(suppl_1): D407-D412.

25. Nagano N, Nakayama N, Ikeda K, Fukuie M, Yokota K, Doi T, et al. EzCatDB: the enzyme reaction database, 2015 update. Nucleic Acids Res 2014, 43(D1): D453-D458.

26. Consortium U. UniProt: the universal protein knowledgebase. Nucleic Acids Res 2018, 46(5): 2699.

27. Berman HM, Westbrook J, Feng Z, Gilliland G, Bhat TN, Weissig H, et al. The protein data bank. Nucleic Acids Res 2000, 28(1): 235-242.

28. MacQueen J. Some methods for classification and analysis of multivariate observations. Proceedings of the fifth Berkeley symposium on mathematical statistics and probability; 1967: Oakland, CA, USA; 1967. p. 281-297.

29. Röblitz S, Weber M. Fuzzy spectral clustering by PCCA+: application to Markov state models and data classification. Advances in Data Analysis and Classification 2013, 7(2): 147-179.

30. Niemyska W, Dabrowski-Tumanski P, Kadlof M, Haglund E, Sułkowski P, Sulkowska Jl. Complex lasso: new entangled motifs in proteins. Sci Rep 2016, 6: 36895.

31. Sulkowska JI. On folding of entangled proteins: knots, lassos, links and $\theta$-curves. Curr Opin Struct Biol 2020, 60: 131-141.

32. Baiesi M, Orlandini E, Seno F, Trovato A. Sequence and structural patterns detected in entangled proteins reveal the importance of co-translational folding. Sci Rep 2019, 9(1): $1-12$.

33. Baiesi M, Orlandini E, Seno F, Trovato A. Exploring the correlation between the folding rates of proteins and the entanglement of their native states. Journal of Physics $A$ : Mathematical and Theoretical 2017, 50(50): 504001.

34. Connolly ML, Kuntz I, Crippen GM. Linked and threaded loops in proteins. Biopolymers: Original Research on Biomolecules 1980, 19(6): 1167-1182.

35. Jarmolinska AI, Gambin A, Sulkowska JI. Knot_pull-python package for biopolymer smoothing and knot detection. Bioinformatics 2020, 36(3): 953-955. 
36. Kröger M. Developments in Polymer Theory and Simulation. Polymers (Basel) 2019, 12(1): 30.

37. Pawlak A. The Entanglements of Macromolecules and Their Influence on the Properties of Polymers. Macromol Chem Phys 2019, 220(10): 1900043.

38. Sułkowska Jl, Sułkowski P, Onuchic J. Dodging the crisis of folding proteins with knots. Proc Natl Acad Sci 2009, 106(9): 3119-3124.

39. Haglund E, Sulkowska JI, Noel JK, Lammert H, Onuchic JN, Jennings PA. Pierced lasso bundles are a new class of knot-like motifs. PLoS Comput Biol 2014, 10(6): e1003613.

40. Haglund E, Sułkowska JI, He Z, Feng G-S, Jennings PA, Onuchic JN. The unique cysteine knot regulates the pleotropic hormone leptin. PLoS One 2012, 7(9): e45654.

41. Lu HP, Xun L, Xie XS. Single-molecule enzymatic dynamics. Science 1998, 282(5395): 1877-1882.

42. Yang H, Luo G, Karnchanaphanurach P, Louie T-M, Rech I, Cova S, et al. Protein conformational dynamics probed by single-molecule electron transfer. Science 2003, 302(5643): 262-266.

43. O'Brien EP, Vendruscolo M, Dobson CM. Kinetic modelling indicates that fast-translating codons can coordinate cotranslational protein folding by avoiding misfolded intermediates. Nat Commun 2014, 5(1): 1-11.

44. Heidary DK, O'Neill JC, Roy M, Jennings PA. An essential intermediate in the folding of dihydrofolate reductase. Proc Natl Acad Sci 2000, 97(11): 5866-5870.

45. Bitran A, Jacobs WM, Zhai X, Shakhnovich E. Cotranslational folding allows misfoldingprone proteins to circumvent deep kinetic traps. Proc Natl Acad Sci 2020, 117(3): 14851495.

46. Porter LL, Looger LL. Extant fold-switching proteins are widespread. Proc Natl Acad Sci 2018, 115(23): 5968-5973.

47. Bryan PN, Orban J. Proteins that switch folds. Curr Opin Struct Biol 2010, 20(4): 482-488.

48. Gaus M, Cui Q, Elstner M. DFTB3: extension of the self-consistent-charge densityfunctional tight-binding method (SCC-DFTB). J Chem Theory Comput 2011, 7(4): 931948. 
bioRxiv preprint doi: https://doi.org/10.1101/2021.08.18.456802; this version posted August 18, 2021. The copyright holder for this preprint

(which was not certified by peer review) is the author/funder, who has granted bioRxiv a license to display the preprint in perpetuity. It is made available under aCC-BY 4.0 International license.

49. Liu Y, Eisenberg D. 3D domain swapping: as domains continue to swap. Protein Sci 2002, 11(6): 1285-1299. 
bioRxiv preprint doi: https://doi.org/10.1101/2021.08.18.456802; this version posted August 18,2021 . The copyright holder for this preprint (which was not certified by peer review) is the author/funder, who has granted bioRxiv a license to display the preprint in perpetuity. It is made available under aCC-BY 4.0 International license.

\section{Graphical Abstract}

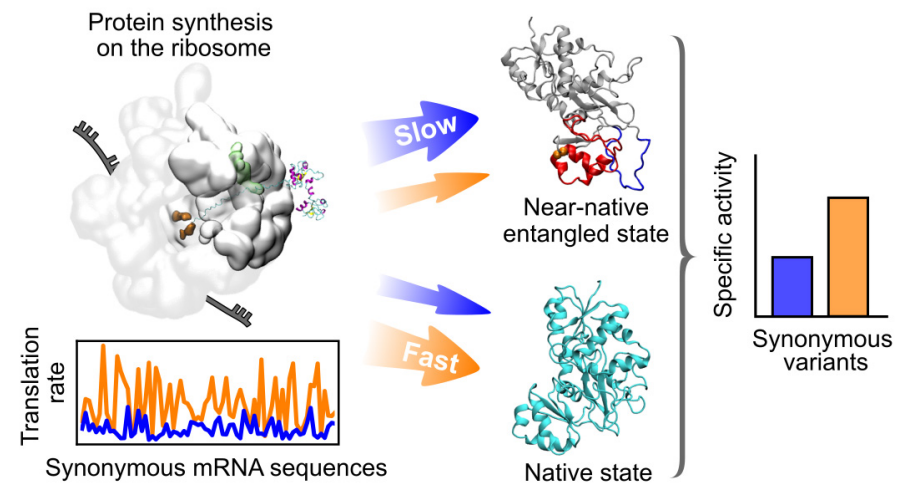

